\title{
A Navigation System for Minimally Invasive CT-Guided Interventions
}

\author{
Markus Nagel ${ }^{1}$, Gerd Schmidt ${ }^{2}$, Ralf Petzold ${ }^{3}$, and Willi A. Kalender ${ }^{1}$ \\ 1 Institute of Medical Physics, University of Erlangen-Nürnberg, Germany \\ markus.nagel@imp.uni-erlangen.de \\ 2 Siemens Medical Solutions, Erlangen, Germany \\ 3 CAS innovations AG, Erlangen, Germany
}

\begin{abstract}
The purpose of our project was to develop a novel navigation system for interventional radiology. Fields of application are minimally invasive percutaneous interventions performed under local anaesthesia. In order to reduce unintentional patient movements we used a patient vacuum immobilization device. Together with the vacuum fixation and a newly developed reference frame we achieved a fully automatic patientto-image registration independent from the tracking system. The combination of the software and a novel designed needle holder allows for an adjustment of the needle within a few seconds. The complete system is adapted to the requirements of the radiologist and to the clinical workflow. For evaluation of the navigation system we performed a phantom study with a perspex phantom and achieved an average needle positioning accuracy of less than $0.7 \mathrm{~mm}$.
\end{abstract}

\section{Introduction}

CT-guided minimally invasive interventions on human subjects are today an established radiological procedure. Such interventions include percutaneous punctures, biopsies, vertebroplasties and radio frequency ablations, as well as therapies requiring the percutaneous advancing of a needle to a definite anatomical position in the patient. CT-guided interventions allow the radiologist to work rapidly with the greatest care and interest of the patient.

The exact placement of the needle in the patient requires a great deal of experience and considerable skills. A limiting factor is the time required to exactly position a needle at the required anatomical site in the patient. Particularly with interventions requiring a high degree of precision, where the incorrect positioning of the needle can lead to life-threatening complications, it is necessary to repeatedly control and correct the position of the advancing needle within the patient using CT control scans. This not only lengthens the entire intervention, but also increases radiation exposure for the patient.

In practice, a number of different auxiliary needle positioning devices are employed. For example, laser targeting devices attached to the gantry or targeting devices which can be fixed directly to the patient 12 . However, none 
of these auxiliary devices permits simultaneous real-time control of the needle, tremble-free needle advancement, and assistance for the implementation of an access path as planned in the patient data. Since the early 1990s, computer and robot-assisted navigation systems have been increasingly used as intervention aids, leading the way for applications in neurosurgery and orthopedics. Applications in the area of interventional radiology are, on the other hand, very rare. The Tirat Hacarmel company utilizes an electromagnetic localization system (UltraGuide1000). This system has already been successfully employed for hepatic and renal punctures, as well as for radio frequency ablations 3. Bale et al. have employed an optical localization system for punctures and radio frequency ablations 4. Medical research shows a continuously growing interest in navigation systems for interventional radiology, as seen by the ever increasing number of groups working on prototypes for needle positioning systems and robotic systems $[5] 6|7| 8]$.

With CT-guided navigation systems, it is first necessary to register the patient according to the images acquired. For this purpose, a fixed reference point, defined by a Dynamic Reference Frame (DRF), is rigidly attached to the patient. For navigation systems used in orthopedics, the DRF is firmly attached to the bones by using surgical screws. For neurosurgical applications, stereotactic frames are attached to the head to allow for the registration of the patient. With minimally invasive interventions such as punctures and biopsies, the rigid attachment of a DRF to the patient represents a problem due to the absence of firm anatomic structures. One solution is to use skin markers which can be positioned in combination with DRFs either directly on the patient's skin, or nearby. However, in this case patient movement would impair the precision of registration or even render the registration useless. Since most minimally invasive interventions are performed under local anesthesia, patient movement is highly probable. A further difficulty with navigation systems is the guidance of the physician during the $3 \mathrm{D}$ orientation of the instruments such as the puncture needle along a planned access path. The orientation process must be fast, precise and simple. In order not to lengthen the entire interventional procedure, the workflow of the navigation system needs to be adapted to the clinical procedure.

We will introduce a new navigation system to solve the problem of patient movement and registration, at the same time enabling the fast, simple and precise orientation of the needle along a planned trajectory. Additionally, the navigation system is ideally matched to the clinical requirements and the clinical workflow.

\section{Materials and Methods}

\subsection{System Overview and Architecture}

The navigation system consists of an optical tracking system (Polaris, NDI, Canada), a standard PC with touch screen as user interface and dedicated navigation software (CAPPA IRad, CAS innovations, Germany), a needle holder, a patient fixation vacuum device (BodyFix ${ }^{\mathrm{TM}}$, Medical Intelligence, Germany) and a reference frame (RF) developed in this work. The RF is attached with 


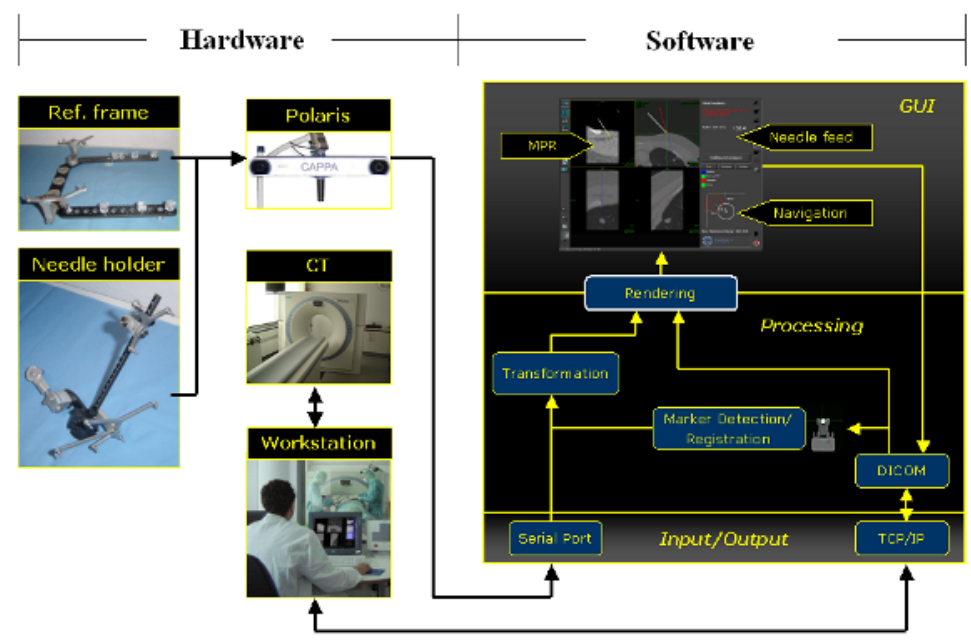

Fig. 1. Overview of the system architecture: CAPPA IRad (Input/Output, Processing, GUI) and hardware (reference frame, needle holder, polaris, CT with workstation)

markers that can be detected automatically in CT images as well as with reflective markers detectable by optical tracking. For image acquisition the navigation system is connected to a CT scanner (Sensation 64, Siemens Medical Solutions, Germany). The system architecture and interface between the components used are illustrated in Fig. 1.

The tracking system is used to localize the position of the reference frame and the needle holder in the operating room. For this purpose, reflecting spheres are fixed with a known geometry on both the reference frame and the needle holder. The position of the needle holder is measured by the tracking system relative to the reference frame in physical space. All necessary 3D coordinates are transmitted from the Polaris to the PC by using the serial port. For the communication of the CAPPA IRad System with the CT scanner we implemented a DICOM network application providing verification, storage and query/retrieve services to enable image transfer using a TCP/IP connection. The DICOM network application is implemented as a background process and set up to receive images as soon as CAPPA IRad is running. After receiving the images, these can be visualized in multi planar reconstruction (MPR) views. We also used the connection to send DICOM images back to the scanner for documentation of performed steps.

\subsection{Description of the System Components and Steps}

Needle holder: The needle holder was constructed such that any medical tool like biopsy syringes or needle hulls can be quickly attached and unmounted. The needle holder itself is mounted at the end of a hydraulic arm. By moving the hydraulic arm (6 DoF) which is mounted on the CT table, the needle holder can be positioned near the patient. Two independent pivot joints on the needle 
holder enable the precise and fast alignment of the needle holder with the planned surgical path, illustrated in Fig. 2 .

Reference frame (RF) and image-to-patient registration: In order to visualize medical instruments like the needle in the $\mathrm{CT}$ images of the patient, an image-to-patient registration is necessary. For that the RF is positioned in proximity to the planned entry point prior to the first CT scan. In addition to the tracking markers, which are used as reference DRF to define the coordinate system of the tracking system, we also placed CT markers in a known geometry on the RF. During image acquisition, all CT markers at the frame must be inside the field of view. After sending the images to the CAPPA IRad system, an integrated marker detection algorithm in the navigation software finds the CT markers in the patient's data set and determines the marker centroids with sub-voxel accuracy. The coordinates of the CT markers in the patient coordinate system and the coordinates of the CT markers in the tracking system are used to derive the registration matrix. After this step, the relation between the images and the patient are fixed.

Patient fixation: We used the BodyFix ${ }^{\mathrm{TM}}$ to fixate the patient to reduce any patient movements relative to the positioned RF. The BodyFix ${ }^{\mathrm{TM}}$ is a double fixation device which consists of a vacuum bag, cushions and a pump. The patient is fitted to the vacuum bag and the cushions are placed on the patient. The air in the bag and in the cushions are exhausted by the pump. In that way the patient is fixed securely to the bag. The fixation device is used successfully in both radiation therapy for the reproducible positioning of the patient for beam delivery [9] and for medical navigation and robotic systems [4].

Calibration of the needle: We implemented and evaluated two kinds of needle navigation possibilities. The first method restricts the adjustment of the needle holder according to the prior planned trajectory without visualizing the length of the needle in the patient's data set. The adjustment of the needle outside the patient is visualized and the software only guides the radiologist to adjust the needle holder. During the needle feed, graduated marks on the needle can be used for depth information.

The second method requires a calibration of the needle to obtain the exact needle length. For that, the radiologist holds the needle tip on a calibration point on the RF. The 3D coordinates of the calibration point are known by the system. A second DRF (needle DRF) is fixed above the needle and calibrated in a way that the origin of the DRF is on top of the needle, as illustrated in Fig. 2, The needle length is defined as the length of the vector between the calibration point and the origin of the needle DRF. During the needle feed, the needle DRF is moved with the needle. The exact position of the needle, especially the tip of the needle, is visible in the patient's data set on the screen.

Software and graphical user interface (GUI): The GUI is designed to allow for an intuitive use of the navigation software. Transfer and loading of the images from CT and registration work fully automatically: The planning module is kept as simple as possible but contains comfortable planning features like oblique 

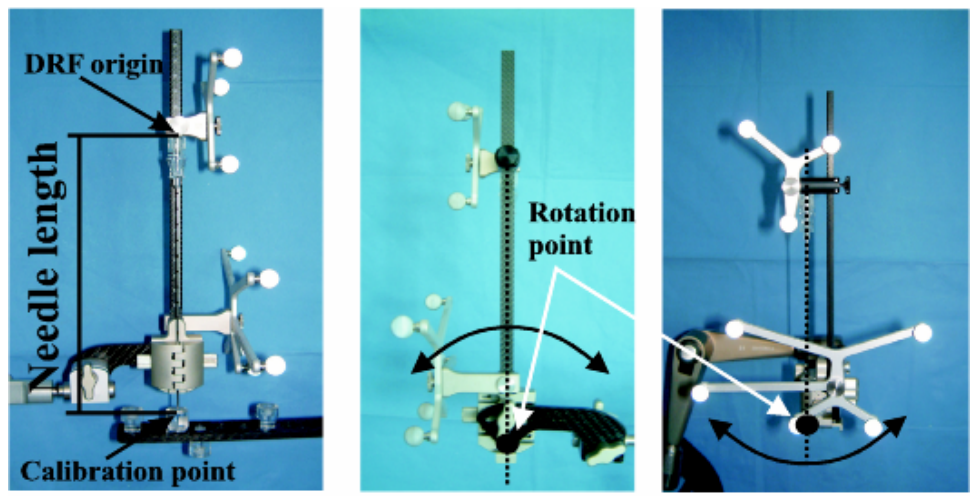

Fig. 2. Left: Calibration step. The length of the needle is calculated from the needle DRF to the calibration point located at the frame. Middle, Right: The needle holder with the two possible movements.

MPRs and accurate planning of trajectories with sub-voxel accuracy. It is also possible to plan arbitrary oblique trajectories. The planning can be performed with the mouse or by touch screen. The combination of the dedicated navigation software and the needle holder allows for an accurate adjustment of the needle according to the planned trajectory within a few seconds. For documentation, screenshots containing information about the final needle position are created, converted to DICOM images and finally sent to the PACS. After the intervention, all images and patient data are removed from the CAPPA IRad system since the local PACS is responsible for archiving and managing the image data.

\subsection{Clinical Workflow}

We analyzed the established clinical workflow of CT-guided, minimally invasive interventions. Based on our investigations we were able to derive the navigation workflow which consists of the following steps:

Preparation of the patient: The patient is placed on the CT bed and immobi-

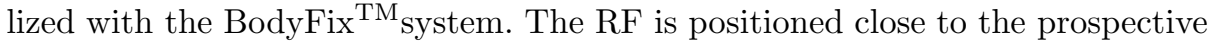
entry point. A field of view is determined to ensure that all CT markers are inside the scanning area.

Importation of scanned images: After scanning, the images are sent from the CT system to the navigation system. All images are internally checked by the software to ensure consistency of patient data before visualization. Additionally, the images are verified by the radiologist and stored on the system to allow for a quick review during the intervention.

Preparation of trajectories: The radiologist defines a trajectory by setting a target and an entry point using the touch screen or the mouse.

Calibration of the needle (optional): The radiologist calibrates the needle used to obtain its length. 
Adjustment of the needle: The adjustment process of the needle with respect to the planned trajectory is divided into two steps. First, the radiologist moves the needle holder in vicinity to the planned entry point, assisted by the navigation system. The next step is the adjustment of the needle holder to adapt the needle axis to the planned trajectory. The software gives the radiologist important information on how to move the needle holder at the two joints to adjust it within a few seconds.

Navigation of needle feed (optional): If the needle was calibrated, the actual position of the needle tip is visible within the CT images of the patient during the needle feed. By extracting information about the location of the needle within the CT coordinates, the system proposes a small region for a control scan in longitudinal direction.

Initiation of control scan: During the needle feed the radiologist is able to initiate control scans and load the images into the navigation system. It is also possible to continue the navigation in the images delivered by the control scan or switch back to the images of the preparation scan.

Documentation: After the needle is at the final position, screenshots can be taken and sent back to the PACS for documentation.

\section{Phantom Test and Results}

To evaluate the navigation system's accuracy we designed a perspex phantom with a base plate including 7 rods with tips, Fig. 3. The phantom was placed at the $\mathrm{CT}$ table and the RF was positioned above the phantom with a distance of $15 \mathrm{~cm}$ from the base plate to the RF. After scanning the phantom we selected the tips of the rods as targets in the planning step of the navigation software and planned trajectories with lengths of $120 \mathrm{~mm}$ and $180 \mathrm{~mm}$.

A standard biopsy needle (18G) was calibrated and adjusted so that the needle tip was exactly on the top of the tips of the rods and the needle was adjusted according to the planned trajectory. The navigation system calculates the vector $\boldsymbol{v}$ between the current needle tip measured by the system and the planned target point and the perpendicular $\boldsymbol{l}$ of the needle path to the target point. The distance $\varepsilon=|\boldsymbol{v}|$ and the perpendicular $\kappa=|\boldsymbol{l}|$ were used to denote the error of the misadjustment. The error includes the error of construction (needle holder and RF), the image-to-patient registration error and the error caused by the tracking system.

For every rod we measured 30 values respectively 15 for both planned trajectory lengths $(120 \mathrm{~cm}$ and $180 \mathrm{~cm})$.

The resulting error $\varepsilon$ of 105 measurements on 7 targets with a trajectory length of $120 \mathrm{~mm}$ was $0.635 \mathrm{~mm}$ rms with $0.228 \mathrm{~mm}$ standard deviation. The length of the perpendicular $\kappa$ at this length was $0.481 \mathrm{~mm}$ with a standard deviation of $0.211 \mathrm{~mm}$. The 105 measurements at the same 7 targets but with a trajectory length of $180 \mathrm{~mm}$ showed nearly the same results. The resulting error $\varepsilon$ was $0.604 \mathrm{~mm}$ rms with $0.217 \mathrm{~mm}$ standard deviation and the perpendicular 


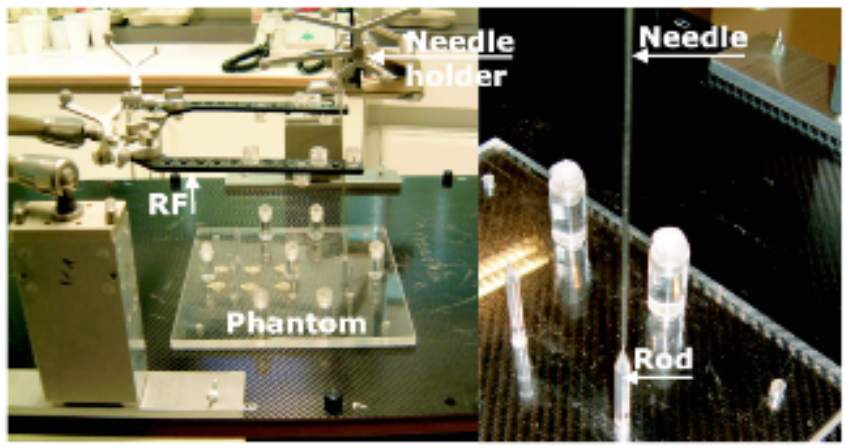

Fig. 3. Perspex phantom study: The needle is exactly adjusted to the planned target (tip of rod) and the needle deviation denoted by the software is used as system adjustment error

Table 1. Results of the phantom study

\begin{tabular}{|l||l|l|l|}
\hline path length & $\mathrm{n}$ & rms $(\varepsilon)$ & rms $(\kappa)$ \\
\hline $120 \mathrm{~mm}$ & 105 & $0.635 \mathrm{~mm} \pm 0.228 \mathrm{~mm}$ & $0,481 \mathrm{~mm} \pm 0.211 \mathrm{~mm}$ \\
\hline $180 \mathrm{~mm}$ & 105 & $0.604 \mathrm{~mm} \pm 0.217 \mathrm{~mm}$ & $0,489 \mathrm{~mm} \pm 0.204 \mathrm{~mm}$ \\
\hline
\end{tabular}

$\kappa$ was $0.489 \mathrm{~mm}$ with a standard deviation of $0.204 \mathrm{~mm}$. All measured values are summarized in Table 1. The maximum measured deviation was $1.2 \mathrm{~mm}$ at a path length of $120 \mathrm{~mm}$.

\section{Discussion}

We presented a new navigation system to be used for minimally invasive percutaneous procedures in interventional radiology. The main focus of the system was to support the radiologist during interventions and to provide a system which is at the same time fully integrated into the clinical workflow. The TCP/IP network connection of the navigation system with the CT scanner and the DICOM protocol enables a manufacturer independent and comfortable image transfer in both directions. By using the developed RF, a fully automatic, tracking system independent patient-to-image registration was achieved. Unpredicted patient movements relative to the RF which could influence the image-to-patient registration are minimized by the fixation. In that way risk related to patient movements was minimal. The calibration allows any needle to be used regardless of the manufacturer. Biopsy syringes or needle hulls can be easily integrated into the system during the intervention. With the combination of software and needle holder it was possible to adjust the needle holder in accordance with a prior planned trajectory. This process was fast and accurate. The needle feed is visualized in the patient's data set to verify the needle placement. This reduces the number of control scans which would otherwise be necessary. In contrast to conventional navigation systems or help devices, our navigation system was 
adapted to the requirements of the radiologist and therefore serves as an ideal tool for various CT-guided percutaneous interventional procedures.

The first accuracy studies with a perspex phantom illustrated a repetitious accuracy of less than $0.7 \mathrm{~mm}$ for two different path lengths $(120 \mathrm{~mm}$ and $180 \mathrm{~mm}$ ). The next step will involve a cadaver study prior to clinical evaluation which has already been approved by an ethics commission.

Acknowledgements. This work was supported by CAS innovations (Erlangen, Germany). The authors are grateful to S. Kurz and S. Wahl for their support during implementation of the complete software. Special thanks go to R. Bale et al. (Sib-Lab, University of Innsbruck, Austria) for helping during the complete development and W. Bautz and M. Uder (Department of Radiology, University of Erlangen-Nürnberg, Germany) for the clinical feedback.

\section{References}

1. Buecker, A., et al.: In: Circular spirit level guidance system for CT- and MR-guided punctures. In Journal of Computer Assisted Tomography 23 (1999) 552-554

2. Frederick, P., et al.: A light-guidance system to be used for CT-guided biopsy. In: Radiology 154 (1985) 535-536

3. Wood, B., et al.: Percutaneous radiofrequency ablation with three-dimensional position sensor guidance. In: Cardiovasc Intervent Radiol (2001) -

4. Bale, R., et al.: A novel vacuum immobilization device and a novel targeting device for computer assisted interventional procedures. In: Proc. of 16th International Congress on "Computer Aided Radiology and Surgery" (2002) 92-97

5. Ra, J., et al.: Spine needle biopsy simulator using visual and force feedback. In: Computer Aided Surgery 7 (2002) 353-363

6. Banovac, F., et al.: Liver tumor biopsy in a respiring phantom with the assistance of a novel electromagnetic navigation device. In: Proc. of Medical Image Computing and Computer-Assisted Intervention, MICCAI (2002) 200-208

7. Cleary, K., et al.: CT-directed robotic biopsy testbed: User interface and coordinate transformations. In: Proc of 15th International Congress on "Computer Aided Radiology and Surgery" (2001) 27-30

8. Megali, G., et al.: A computer-assisted robotic ultrasound-guided biopsy system for video-assisted surgery. In: Proc. of Medical Image Computing and ComputerAssisted Intervention, MICCAI (2003) 343-350

9. Fuss, M., et al.: Repositioning accuracy of a commercially available double-vacuum whole body immobilization system for stereotactic body radiation therapy. In: Technology in Cancer Research and Treatment (2004) 59-68 